Dobell, A. R., and Y. C. Ho

"Optimal Investment Policy: An Example of a Control Problem in Economic Theory." IEEE Transactions on Automatic Control 12.1(1967): 4-14.

Reprinted with permission from The Institute of Electrical and Electronics Engineers, Inc. 


\title{
Optimal Investment Policy: An Example of a Control Problem in Economic Theory
}

\author{
A. R. DOBELL AND Y. C. HO, SENior MEMber, IEEE
}

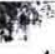 \\ T}

Abstract-A problem in mathematical economics concerning the optimal investment of resources is solved via the techniques of optimal control theory. Interesting theoretical complications include the simultaneous presence of interdependent control variable inequality constraints, state variable inequality constraints, and singularity conditions. Economic implications of the results are briefly discussed.

\section{INTRODUCTION} THERE has been, in economic theory, extensive discussion of aggregate models of capital accumulation, much of it directed toward the determination of investment plans optimal under some specified criterion [3], [6], [8], [9]. This problem may be formulated as one in optimal control. The following example, involving two state variables rather than the singlestate variable customary in earlier analyses, has been artificially simplified in other respects in order to highlight the way in which newly developed techniques of control theory enter into the solution of such problems in economic growth. Discussion of a more general formulation of the economic model will be undertaken elsewhere.

Essentially, the economic problem is in the allocation of output between consumption and investment, and in the composition of investment, which may be directed toward growth in alternative capital assets. Thus, it is supposed that the capital stock and the labor force of the economy at any moment determines the maximum output flow; then, by its expenditures on capital accumulation and labor training, the society determines the rates of growth of capital and trained labor, and hence determines the paths of output, consumption, and employment for the future. One possible measure of the resulting current welfare in the society is per capita consumption.

In Section II the problem is formally posed. Some relevant facts from control theory are summarized in

Manuscript received April 22, 1966; revised October 14, 1966. The work reported in this paper was supported in part by the Harvard University Program on Technology and Society under a longterm grant from the International Business Machines Corporation; and in part under the Joint Services Electronics Contract NONR 1866 (16). Responsibility for the views expressed rests solely with the authors.

The authors are with Harvard University, Cambridge, Mass.
Section III, and a solution is given in Section IV. A brief economic interpretation of some of the optimality conditions, and some concluding comments, are set out in Section V.

\section{The Problem}

The allocation problem is formulated in this section. It is assumed that the total value of the community's annual production of goods can be represented as a function of its capital stock and employed labor force. Thus, letting $Q$ (measured in dollars per year) denote the value of annual production, $K$ (measured in dollars) the value (replacement cost) of the physical capital stock, and $W$ (measured in men) the number of employed workers, the assumption is that

$$
Q=F(K, W) .
$$

The function $F$ is assumed to be homogeneous of degree one in $K$ and $W$, to be positive, with positive first partial derivatives, concave in $K$ and $W$, and to satisfy

$$
F(0, W)=F(K, 0)=0 .
$$

Moreover it is assumed that a point $(K, W)$ can be found which satisfies the equation

$$
\frac{\partial F}{\partial K}(K, W)=n+\gamma+\delta
$$

for all relevant values of the parameters $n, \gamma, \delta$, to be introduced below. (All variables are functions of time, but the argument $t$ will generally be omitted.)

Two categories of nonconsumption use of the output flow represented by $Q$ are recognized in this analysis. The first is allocation of part of output to accumulation of further capital stock. Let $I$ (measured in dollars per year) be the rate of flow of output directed toward such additions to capital; $I$ is then the rate of gross investment in physical capital. The realized net addition to physical capital is, however, not so large, since some investment is necessary simply to maintain the capital stock at existing levels. Approximating this replacement 
requirement by $\delta K$ (dollars per year), ${ }^{1}$

$$
I=K+\delta K, \text { or } \quad K=I-\delta K .
$$

(This equation, thus, says simply that the gross investment rate $I$ has two components - the portion required to match retirement of existing capital and the part left over for increase of the stock.)

A second category of expenditure arises because it is assumed that training costs are incurred in qualifying labor for entry into production. For the present work it will be assumed that this cost is a constant, $d$ dollars per man, ${ }^{2}$ and therefore that for every man newly trained in a given year, the consumption of the community must be reduced by $d$ dollars that year. Let it be supposed that the number of men which it is necessary to train in any year in order to maintain the level of employment constant at $W$ is $\mu W$ per year. Then, since $\dot{W}+\mu W$ is the total number of men newly trained per year, and $d$ is the cost per man, the reduction in attainable consumption due to training of new labor may be written

$$
E=d(W+\mu W), \text { or } \quad W=E / d-\mu W .
$$

The variable $E$ might then be called the rate of gross investment in "human capital," and (3) shows that it also has two components representing provision for retirement of existing stocks and provision for increase of stocks.

Consumption (in dollars per year) is given as the residual

$$
\begin{aligned}
C & =F(K, W)-I-E \\
& =F(K, W)-(\dot{K}+\delta K)-d(W+\mu W) .
\end{aligned}
$$

This equation thus reflects the fact that the total output flow $Q$ is allocated among three uses-a flow $C$ destined for present consumption, a flow $I$ representing

${ }^{1}$ The noneconomist should be warned that this discussion glosses over some difficulties in valuation of capital. Here these are avoided by measuring capital stock in dollars and output in dollars per year, and supposing that the physical unit of capital stock and the sacrifice of consumption required to acquire one physical unit are unchanging. More precise discussion of this issue would be long, and not really relevant to the concerns of this paper.

A word about the depreciation assumption is also appropriate. Throughout, the assumption that the proportion of the capital stock and of the labor force being retired each year is a constant is rather unsatisfactory in a dynamic growing economy, except as a crude approximation. Simple dependence on time or on the state of the system could easily be admitted, but it should be observed that the most appealing hypothesis-that the probability of retirement for a machine or a laborer depends on the length of time already servedwould prohibit any finite representation of the state of the system. The reason is that the requirement for replacement, and hence the rate of increase, of both physical capital and labor would then depend on the present age structure of each, rather than just the total stock, and thus would depend on the entire past histories of investment in capital and labor. The "exponential decay" assumption avoids this difficulty.

${ }^{2}$ Alternatively, if the training cost $d$ is assumed to depend upon $K / W$ (the capital per worker) at the time of training, or upon $W / L$ (the employment rate) at the time of training, the analysis carries through. In either of these cases there arises the possibility of optimal balanced growth at less than full employment. the consumption foregone because of the use of resources in adding to stocks of physical capital, and a flow $E$ representing the consumption foregone in order to devote resources to training newly employed labor.

The development of this model economy is completely determined when, from a given initial position, the present and future allocation of output to the two investment uses (physical capital and labor training) is specified. Supposing this to be open to choice, one may ask what allocation would be best for an economy with given production possibilities. The criterion for the present problem will be taken to be

$$
J=\int_{0}^{T} \frac{C(t)}{L(t)} \exp (-\gamma t) d t
$$

where $T$ is a "planning horizon" which may be infinite, $\gamma$ is a rate of discount of future flows, and $L$ represents the total labor force. A given allocation plan, resulting in a unique consumption path, thus yields a single value for $J$. The plan yielding the greatest such value will be selected as best.

A feasible allocation must guarantee that none of the flows $C, I, E$ become negative, reflecting the fact that investment cannot be financed by permitting consumption to fall below zero (defined to be the subsistence level), and that resources once embodied in machines or training cannot be recovered and transferred to provide consumption, nor transformed into one another. This requirement can be expressed conveniently by defining two gross investment rates as proportions of total output $Q$,

$$
s=\frac{I}{Q}=\frac{\dot{K}+\delta K}{F(K, W)}, \quad e=\frac{E}{Q}=\frac{d(W+\mu W)}{F(K, W)}
$$

and requiring

$$
0 \leq s, \quad 0 \leq e, \quad s+e \leq 1,
$$

the need for the last inequality stemming from the fact that

$$
C=(1-s-e) F(K, W)
$$

and $C$ is required to be non-negative.

There is a further constraint, arising from the fact that labor is not produced within the system. Here it is assumed that the total labor force is determined exogenously according to the simple growth law

$$
L=n L, \quad L(0)=L_{0}
$$

where $n$ is a constant rate (per year) of labor force growth. Then the number of employed workers must satisfy the full employment constraint

$$
W \leq L
$$

Two treatments of this restriction are possible. The first is simply to incorporate the hard barrier of full employment in to the analysis by adding conditions (9) and (10) 
to (6) and (7). This form of the problem, which presents the greater technical challenge, will be discussed here. An alternative is to argue that the pressures of full emplyment come into play more smoothly, with mounting social costs associated with rising employment rates beyond some level. In this case, a penalty cost function might be constructed to represent the social costs of inflationary pressures. There is no need to discuss this latter approach explicitly here. ${ }^{3}$

If the horizon $T$ is infinite, the terminal conditions may be left free, with the analysis itself determining the optimal endpoint. But, as is plausible - and recognized in the economics literature [3] - for a finite horizon problem, terminal conditions are essential to represent any weight attached to the welfare (so far as capital stocks are relevant) of generations beyond the horizon. Terminal targets may be expressed as

$$
\begin{aligned}
& W(T) \geq W_{T}, \quad \text { or } \quad \frac{W(T)}{L(T)} \geq w_{T} ; \\
& K(T) \geq K_{T}, \quad \text { or } \quad \frac{K(T)}{L(T)} \geq r_{T} .
\end{aligned}
$$

Specification of the system is thus complete. It is convenient, however, to rewrite the problem in terms of the capital-labor ratio and the employment rate, as is permitted by the assumption of linear homogeneity of the function $F$. Writing the capital-labor ratio and the employment rate as

$$
r=\frac{K}{L} \quad \text { and } \quad w=\frac{W}{L}
$$

respectively, and introducing the function $f(r)$ to represent $F(r, 1)$, the criterion becomes

$$
J=\int_{0}^{T}(1-s-e) w f(r / w) \exp (-\gamma t) d t .
$$

The growth of the system is given by two equations which are obtained by observing that

$$
\begin{aligned}
\dot{r} & =\left(\frac{K}{K}-\frac{L}{L}\right) \frac{K}{L}=\frac{K}{L}-n r \\
\dot{w} & =\left(\frac{W}{W}-\frac{L}{L}\right) \frac{W}{L}=\frac{W}{L}-n w
\end{aligned}
$$

${ }^{3}$ Introducing the employment rate $w=W / L$, and a nominal full employment level $\bar{w}<1$, one computationally convenient form would be

$$
P(t)=\frac{A}{2}(w-\bar{w})^{2} U(w-\bar{w})
$$

where $A$ is a constant and $U$ is a unit step function. The criterion is then modified to

$$
J=\int_{0}^{T}\left(\frac{C}{L}-P\right) \exp (-\gamma t) d t .
$$

Analysis of this problem was completed before solution of the inequality form, and it was later verified that as $A \rightarrow \infty, \bar{w} \rightarrow 1$, the solution goes over to that given in this paper for the inequality form. and using (6) to substitute for $\dot{K}$ and $\dot{W}$. The result is

$$
\begin{aligned}
& \dot{r}=\operatorname{swf}(r / w)-(n+\delta) r \\
& \dot{w}=\frac{e}{d} w f(r / w)-(n+\mu) w .
\end{aligned}
$$

Thus the problem, finally, is to determine the investment policy $s(t), e(t)$, which yields a maximum to $J$, subject to the system equations (13) and (14), the constraints (7), (8), (9), and (10), and the terminal conditions (11). A solution is outlined in Section IV, after a summary of some facts from control theory.

\section{Facts From Control Theory}

Before attempting the solution of the problem formulated above, it is appropriate to summarize in one place a set of recently developed control theoretic results scattered till now in different places in the literature. Consider the dynamic system

$$
\dot{x}=f(x, u, t) ; \quad x\left(l_{0}\right)=x_{0}
$$

where $\mathrm{x}$ is an $n$-vector of state variables, $\boldsymbol{u}$ an $m$-vector of control variables, and $f$ an $n$-vector function of the variables $\mathrm{x}, \boldsymbol{u}$, and $t$, of sufficient smoothness to insure unique solution of the differential equation (15) for all initial values $x_{0}$ that may be of interest. The problem is to choose the control function $\boldsymbol{u}(t) \quad 0 \leq t \leq T$ such that the criterion

$$
J=\int_{0}^{T} L(x, u, t) d \ell
$$

is maximized subject to the constraints

$$
\downarrow(x(T), T)=0 \quad \begin{aligned}
& \text { (q equations of terminal con- } \\
& \text { straints) }
\end{aligned}
$$

$\boldsymbol{u}(l) \in U$

( $U$ is a convex set in $R^{m}$; inequality constraints on the control variables)

$C(x, t) \leq 0$

(scalar inequality constraint on the state variables)

where it is again assumed that the various functions $L$, ษ, $C$ are sufficiently smooth to insure the existence of partial derivatives of various order that may be required in the ensuing analysis. Defining for convenience the scalar function

$$
H(x, \lambda, u, t)=L+\lambda^{T} f,
$$

the necessary conditions for the optimality of the control function $\boldsymbol{u}(t)$ can now be stated as follows. The optimal $\boldsymbol{u}^{0}(t)$ must necessarily satisfy

i) Euler-Lagrange equations when $C(x, t)<0$ [7]

$$
\dot{\mathbf{x}}=\boldsymbol{f}\left(\mathrm{x}, \boldsymbol{u}^{0}, t\right) ; \mathrm{x}\left(t_{0}\right)=\mathrm{x}_{0}, \quad \boldsymbol{\psi}(\mathrm{x}(T), T)=0
$$

4 The notation $f_{x}$ denotes the vector of first partial derivatives of the function $f$ with respect to the components of the vector $\mathrm{x}$; similarly $f_{x}$ denotes the matrix of the partial derivatives. 


$$
\begin{array}{ll}
\dot{\lambda}^{T}=-H_{x}=-\lambda^{T} f_{x}-L_{x} & \lambda^{T}(T)=\left.v^{T} \mho_{x}\right|_{x(T)} \\
H\left(x, \lambda, u^{0}, t\right) \geq H(x, \lambda, u, t) & u \in U
\end{array}
$$

i.e., $\boldsymbol{u}^{0}$ is determined by maximizing the Hamiltonian function $H$ for fixed $x$ and $\lambda$; the vector $v$ is a $q$-vector of constants to be chosen appropriately.

ii) Euler-Lagrange equations when $C(x, t)=0$ [1], [7]

In this case, (20) and (22) are to be modified by

$$
\begin{aligned}
& H(x, \lambda, u, \eta, t) \equiv L+\lambda^{T} f+\eta C \\
& \dot{\lambda}^{T}=-H_{x}=-\lambda^{T} f_{x}-L_{x}-\eta(C)_{x}
\end{aligned}
$$

where $\eta(t)$ is a scalar function of time satisfying

$$
\begin{gathered}
\eta(t) \leq 0, \\
\frac{\partial H}{\partial u_{i}}=L_{u_{i}}+\lambda^{T} f_{u_{i}}+\eta(C)_{u_{i}}=0,
\end{gathered}
$$

and where $u_{i}{ }^{0}$ is determined by

$$
C=C_{\mathrm{x}} f+C_{\imath}=0
$$

provided $u_{i}{ }^{0} \in U$, and all other $u_{j}{ }^{0} j \neq i$ are determined by maximizing the Hamiltonian function subject to $u^{0} \in U$.

\section{iii) Corner conditions}

It is assumed that the optimal trajectory does not initially lie on the constraint $C=0$. Let $t$ be the time when the constraint $C \leq 0$ changes from a strict inequality to an equality; then at $t$

$$
\begin{aligned}
& \lambda^{T}\left(t^{-}\right)=\lambda^{T}\left(t^{+}\right)+\left.\pi C_{x}\right|_{x(t)} \\
& H\left(t^{-}\right)=H\left(t^{+}\right)-\pi C_{t}
\end{aligned}
$$

and

$$
C(x(t), t)=0
$$

where $\pi$ is an appropriately chosen constant.

\section{iv) Singularity conditions [5], [12]}

In i) and ii), if the maximization of the Hamiltonian function leads to nonunique determination of one or more control variables $u_{j}$, then a "singular" situation in $u_{j}$ results. E.g., if $H=g(x, \lambda, t) u_{j}+$ terms involving no $u_{j}$, then it is possible for solutions of (21) and (22) or $\left(22^{\prime}\right)$ to take on values such that $g(x, \lambda, t)=0$ for a finite length of time. In this case, $H$ is independent of $u_{j}$ and maximization does not determine the optimal value of $u_{j}$. In such singular cases, the control variables $u_{j}$ are determined by differentiating the condition for singularity (i.e., $g=0$ ) with respect to time twice

$$
\begin{aligned}
& \frac{d}{d t} g \equiv \frac{d}{d t}\left(\frac{\partial}{\partial u_{j}} H\right)=0 \\
& \frac{d^{2}}{d t^{2}}\left(\frac{\partial}{\partial u_{j}} H\right)=0 .
\end{aligned}
$$

Equation (31) in general will involve $u_{j}$ explicitly and can be used for the unique determination of $u_{j}{ }^{0}$ (pro- vided $u_{j}{ }^{0} \in U$ ) which is in turn required by (21) and (22) or $\left(22^{\prime}\right)$ in order to calculate the particular $x(t)$ and $\lambda(t)$ which satisfy (30) and $g=0$. In other words, (31) in this case plays a role analogous to (23) or (26) in supplying the values of the control variables for (21) and (22). with (30) and $g=0$ serving as extra constraints on the possible trajectories of $\mathrm{x}(t)$ and $\lambda(t)$. Furthermore, on these "singular" trajectories, it is necessary to have

$$
\frac{\partial}{\partial u_{j}} \frac{d^{2}}{d t^{2}}\left(\frac{\partial}{\partial u_{j}} H\right) \geq 0 .
$$

\section{Remarks}

a) Statement i) represents the usual statement of the Maximum Principle [7] and leads to the usual two-point boundary value problem as defined by (21)-(23). If using (23) is considered to eliminate the variables $u$ completely, then at $t=0$ there are $n$ unknowns, $\lambda(0)$, and at $t=T, 2 n+q$ unknowns $\mathrm{x}(T), \lambda(T), \boldsymbol{v}$, and $n+q$ conditions.

b) There are at least two approaches [1], [7] to the handling of state variable inequality constraints $C(x, t)$ $\leq 0$, both involving the modification of the definition of the Hamiltonian function and the Euler equation. The approach in ii) follows Bryson-Denham-Dreyfus. For the connection with that of Pontriagin-Gamkrelidze, see [4].

c) It is tactily assumed that (26) will always lead to values of $u_{i}$ which are realizable. In other words, cases are explicitly ruled out where the specification of $U, C$, and $x_{0}$ lead to impossible situations. On the other hand, if (31) results in $u_{j}$ which violates $u_{j} \in U$, then it merely implies that the singular conditions are no longer sustainable.

d) Note that the corner condition (29) at $t$ and (26) insure that $C(x, t)=0$ continuously when Case ii) is in effect.

e) Equation (32) is known as the generalized Legendre-Clebsch condition due to its resemblance to $\partial / \partial u_{j}\left(\partial H / \partial u_{j}\right) \geq 0$ for the nonsingular case.

f) The statements ii) and iv) are not the most general ones possible. In particular, it may happen that (26) or (31) are independent of $u_{i}$ or $u_{j}$ in which case further manipulations are necessary. For details see [2], [5], [11]. The present statements are sufficient for the purpose of this paper; proofs of all can be found in the appropriate references.

g) Note that the singular Case iv) has to be considered whenever the control variables enter into the system differential equations and the criterion function linearly. This happens to be the case for the economic optimization problem here. Furthermore, it will be shown that these happen to be economically the most interesting cases. 


\section{Outline Solution ${ }^{5}$}

The problem, it will be recalled, is to find the investment allocation plan which yields a maximum of

$$
J=\int_{0}^{T}[1-s-e] w f \exp (-\gamma t) d t
$$

subject to

$$
\begin{aligned}
& \dot{r}=s w f-(n+\delta) r \quad r(0)=r_{0} \\
& \dot{w}=\frac{e}{d} w f-(n+\mu) w \quad w(0)=w_{0} \\
& 0 \leq s, \quad 0 \leq e, \quad 0 \leq s+e \leq 1 \\
& 0 \leq w \leq 1, \quad 0 \leq r
\end{aligned}
$$

and to terminal conditions

$$
r(T)=r_{T}, \quad w(T)=w_{T} .
$$

\begin{tabular}{|c|c|c|}
\hline & General Problem & Economic Problem \\
\hline State variables & $x$ & $\begin{array}{l}r \\
w\end{array}$ \\
\hline Control variables & $u$ & $\begin{array}{l}s \\
e\end{array}$ \\
\hline System function & $f$ & $\begin{array}{c}\operatorname{swf}\left(\frac{r}{w}\right)-(n+\delta) r=\dot{r} \\
\frac{e}{d} w f\left(\frac{r}{w}\right)-(n+\mu) w=w \dot{v}\end{array}$ \\
\hline Criterion & $L$ & $\begin{aligned}(1-s-e) w f\left(\frac{r}{w}\right) \\
\cdot \exp (-\gamma t)\end{aligned}$ \\
\hline Control constraints & $U\left(\begin{array}{c}\text { Convex set } \\
\text { in } R^{m}\end{array}\right)$ & $\begin{array}{l}s \geq 0 ; \quad e \geq 0 \\
1 \geq s+e\end{array}$ \\
\hline Terminal constraints & $\psi(x(T), T)=0$ & $\begin{array}{l}\quad r(T)=r_{T} ; w(T)=w_{T} \\
\text { or } r(T), w(T) \text { free }\end{array}$ \\
\hline $\begin{array}{c}\text { State variable } \\
\text { constraints }\end{array}$ & $C(\mathrm{x}, t) \leq 0$ & $w(t) \leq 1^{*}$ \\
\hline
\end{tabular}

These terminal conditions may subsequently be weakened or removed.

\section{A. Catalogue of Cases}

It is clear that this problem fits into the class of control problems covered by i)-iv) in the previous sections. The following table identifies the specific variables of this problem with those of the general problem.

* Strictly speaking, constraints $r \geq 0, w \geq 0$ should also be included. However, it turns out that they are not effective for the problem of interest.

5 Throughout the following, the function $f$, appearing without specific argument, is to be understood as denoting $f(r / w)$. The argument $r / w$ will be omitted. Further, the subscripts $\lambda_{r} \lambda_{w}$ denote components of the vector $\lambda$ of auxiliary variables, and do not indicate partial derivatives.
Define

$H=(1-s-e) w f \exp (-\gamma t)+\lambda_{r}[s w f-(n+\delta) r]$

$$
+\left(\lambda_{w}+\eta\right)\left[\frac{e}{d} w f-(n+\mu) w\right] .
$$

Then the Euler-Lagrange equations are accordingly

$$
\begin{gathered}
\dot{\lambda}_{r}=-H_{r}=-\left[(1-s-e) \exp (-\gamma t)+s \lambda_{r}\right. \\
\left.+\frac{e}{d}\left(\lambda_{w}+\eta\right)\right] f^{\prime}+(n+\delta) \lambda_{r} \\
\dot{\lambda}_{w}=-H_{w}=-\left[(1-s-e) \exp (-\gamma t)+s \lambda_{r}\right. \\
\left.+\frac{e}{d}\left(\lambda_{w}+\eta\right)\right]\left(f-\frac{r}{w} f^{\prime}\right)+(n+\mu)\left(\lambda_{w}+\eta\right)
\end{gathered}
$$

where

$$
\eta=\left\{\begin{aligned}
0 & \text { if } w<1 \\
\leq 0 & \text { if } w=1
\end{aligned}\right.
$$

The interesting cases appear when the maximization of the Hamiltonian function is attempted. Note that the control variables enter into $H$ linearly. Hence, in general, the maximum should occur on the boundary of the constraints of the control or state variables. The geometrical situation of the maximization for the case $w<1$ is illustrated in Fig. 1 in terms of the admissible region of $s$ and $e$ and the vector

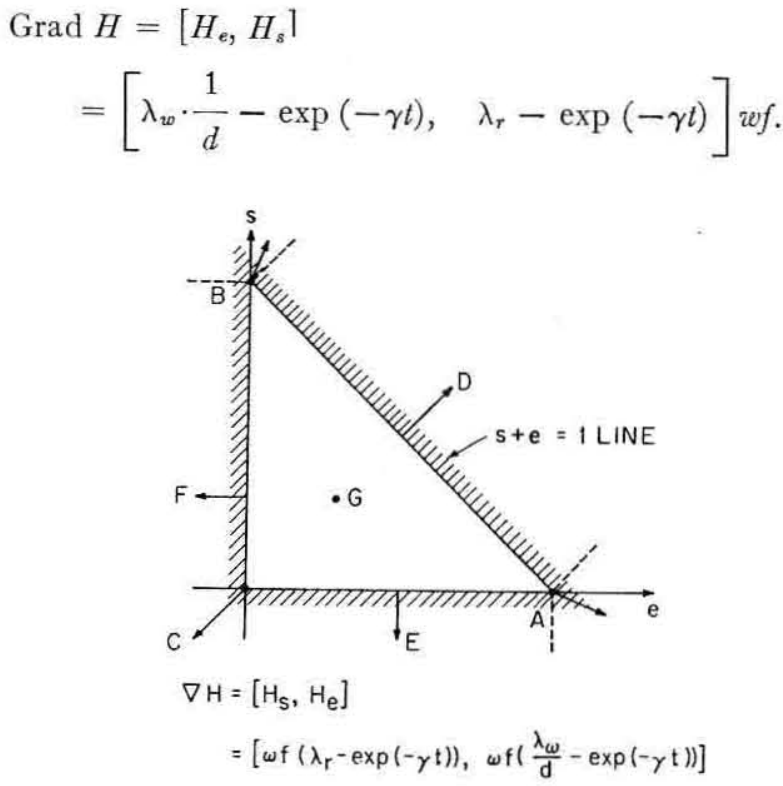

Fig. 1. Control space and possible directions of $\nabla H$.

The seven vectors show the seven possible directions of the Grad $H$ vector (Case G corresponds to the case Grad $H=0$ ) and the respective value, or values, of the maximizing control variables $s$ and $e$. For Cases A, B, and $\mathrm{C}$ the choices for $s$ and $e$ are obvious. The trajectories of the system (solutions of the system and EulerLagrange equations) can be readily determined by substituting the appropriate values of $s$ and $e$ into (34), 
(38), and (39). The remaining four Cases D through $\mathrm{G}$ represent singular cases and require somewhat more anaysis, as follows.

Case $D$ : This case is defined by the condition

$$
H_{s}-H_{e}=0 ; \quad H_{s}=H_{e}>0
$$

which implies $s+e=1$ and

$$
\lambda_{r}=\frac{\lambda_{w}}{d}
$$

along a singular trajectory. Differentiating (43) with respect to time [see (30)], the following equation is obtained:

$$
f-\frac{r}{w} f^{\prime}-d f^{\prime}=(\mu-\delta) d .
$$

One more differentiation of (44) leads to [see (31)]

$$
f\left(s w-e \frac{r}{d}\right)-(\delta-\mu) r=0,
$$

from which,

$$
\begin{aligned}
& s=\frac{w f-(\delta-\mu) r}{f\left(w+\frac{r}{d}\right)} \\
& e=\frac{f \frac{r}{d}+(\delta-\mu) r}{f\left(w+\frac{r}{d}\right)} .
\end{aligned}
$$

Equation (46) can then be used to specify differential equations for $r, w, \lambda_{r}, \lambda_{w}$.

Case $E$ : This case is defined by

$$
\begin{aligned}
& H_{s}<0 \Rightarrow s=0 \\
& H_{e}=0 \Rightarrow \lambda_{w}=d \exp (-\gamma t) .
\end{aligned}
$$

Hence,

$$
\dot{\lambda}_{w}=-d \gamma \exp (-\gamma t)
$$

which implies

$$
f-\frac{r}{w} f^{\prime}=(n+\mu+\gamma) d .
$$

Further differentiation yields

$$
\begin{aligned}
& e=\frac{d(\mu-\delta)}{f} \\
& s=0 .
\end{aligned}
$$

Case $F$ : Here the condition is

$$
\begin{aligned}
& H_{e}<0 \Rightarrow e=0 \\
& H_{s}=0 \Rightarrow \lambda_{r}=\exp (-\gamma t) .
\end{aligned}
$$

This leads, upon differentiation, to

$$
f^{\prime}=n+\delta+\gamma
$$

which, in turn, requires

$$
s=\frac{(\delta-\mu) r}{w} .
$$

Observe that, depending on the sign of $(\mu-\delta)$, one of $\mathrm{E}$ or $\mathrm{F}$ is not a sustainable path. Henceforth, it shall be assumed that $\mu>\delta$.

Case $G$ : This case requires

$$
H_{e}=H_{s}=0
$$

so that,

$$
f^{\prime}=n+\delta+\gamma
$$

and

$$
\left(f-f^{\prime} \frac{r}{w}\right)=(n+\mu+\gamma) d .
$$

These two conditions are in general satisfied by different values of $r$ and $w$ so that Case $\mathrm{G}$ is not a sustainable path.

Now the different cases corresponding to $w(t)$ $=1 \quad(\eta<0)$ will be treated. Equation (26) becomes simply $\dot{w}=0$ which implies

$$
e=\frac{(n+\mu) d}{f} \quad \text { [Compare (26)] }
$$

and (25) determines $\eta$ as

$$
\eta(t)=d \exp (-\gamma t)-\lambda_{w} .
$$

Three more cases are possible from a consideration of maximization of $H$ with respect to the second control variable $s$.

Case 1:

$$
H_{s}>0 \text {. }
$$

One has

$$
s=1-e=\frac{f-(n+\mu) d}{f}
$$

(this being the largest value which can be taken without violating the constraint $s+e \leq 1$ ) and the motion is determined.

Case 2:

$$
H_{s}<0 .
$$

One has $s=0$ and the motion is again determined.

Case 3: $H_{8}=0$ corresponding to a singular situation on the constraint $w=1$. Then

$$
\lambda_{r}=\exp (-\gamma t)
$$

which implies through differentiation

$$
f^{\prime}=n+\gamma+\delta
$$


and

$$
s=\frac{(n+\delta) r}{f} .
$$

These particular values for $s$ and $e$ result in $\dot{r}=\dot{w}=0$ and yield the equilibrium state for the economic system.

\section{Remarks}

h) In terms of the geometrical picture of Fig. 1, Cases 1-3 can be visualized by considering the vectors Grad $H$ and

$$
\begin{aligned}
\operatorname{Grad} H^{*} \equiv & {\left[H_{e}{ }^{*}, H_{s}\right] } \\
& \equiv\left[\frac{\lambda_{w}}{d}-\exp (-\gamma t), \lambda_{r}-\exp (-\gamma t)\right]
\end{aligned}
$$

where $H_{e}{ }^{*}$ is the change in $H$ due to change in $e$ if one ignores the presence of the constraint $w=1$. This is shown in Fig. 2. The meaning of the multiplier function $\eta(t)$ is now clear. It is adjusted to ensure that $H_{t}=0$ on the constraint boundary. Furthermore, $H_{e}{ }^{*}$ always points in the direction of violation of the constraint boundary which explains the sign of $\eta(t)$. Note that in the control space the constraint corresponding to $w \leq 1$ is not fixed and depends on the values of the state variables $r$ and $w$.

i) It can be verified that the generalized LegendreClebsch condition is satisfied for all singular Cases $E$, $D$, and 3 . For details see Appendix I.

This lengthy catalogue of cases gives all possible modes of movement for the system. The resultant system of equations for each case may be easily derived.

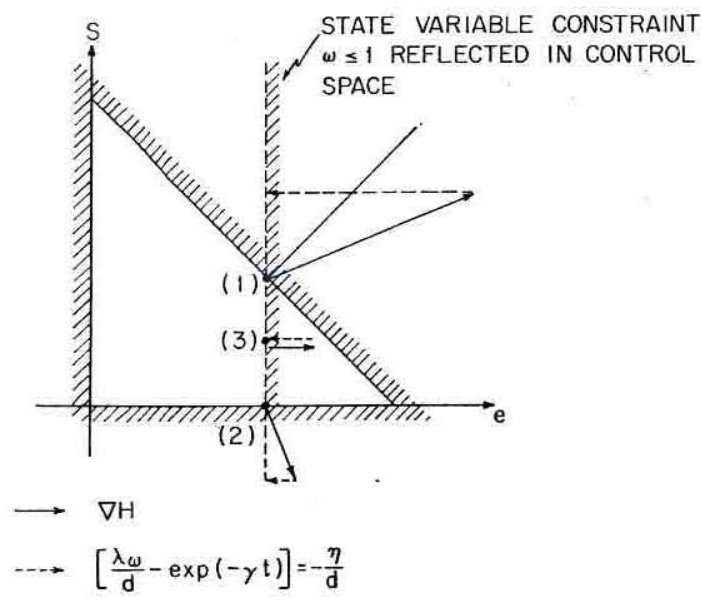

Fig. 2. Cases on state variable constraint boundary.

B. Corner Conditions, Terminal Conditions, and Transition Sequences

The remaining problem is to determine the sequence of cases (i.e., to piece together a trajectory out of the individual motions of the differential equations governing each case) appropriate for the given initial condition

$$
\begin{aligned}
& r(0)=r_{0} \\
& w(0)=w_{0},
\end{aligned}
$$

the necessary terminal conditions and intermediate corner conditions. The corner conditions occur at the time of entry $t_{1}$ onto the constraint boundary $w=1$. They are [cf., (27)-(29)]

$$
\begin{aligned}
w\left(t_{1}\right) & =1 \\
\lambda_{w}\left(t_{1}^{-}\right) & =\lambda_{w}\left(t_{1}^{+}\right)+\pi \\
\lambda_{r}\left(t_{1}^{-}\right) & =\lambda_{r}\left(t_{1}^{+}\right) .
\end{aligned}
$$

The necessary terminal conditions depend upon the terminal constraint imposed. Two cases are considered.

Terminal state and time fixed: From (22) one has

$$
\begin{gathered}
r(T)=r_{T} \\
w(T)=w_{T} \\
\lambda_{r}(T)=\nu_{r}=\text { constant to be determined } \\
\lambda_{w}(T)=\nu_{w}=\text { constant to be determined. }
\end{gathered}
$$

Terminal time infinite and states free: In this case,

$$
\begin{aligned}
& \lambda_{r}(\infty)=0 \\
& \lambda_{w}(\infty)=0 .
\end{aligned}
$$

It is readily verified that this case can only be realized via the equilibrium Case 3 . This is a standard result for models of optimal saving (see [3], [10]).

Finally, the possible transition from case to case must be considered. In this connection, it may be noted that both $H_{e}$ and $H_{s}$ are continuous functions of time (except at the entry corner $t_{1}$ ). This fact helps to eliminate certain transition sequences. Figure 3 lists the various possible transitions. ${ }^{6}$ One type of transition, that of getting off the constraint $w=1$, deserves further discussion. Referring to Fig. 2 once again, note that the transition from Case 1 to $B$ occurs whenever

$$
\lambda_{r}-\exp (-\gamma t)>\left|\frac{\eta}{d}\right|,
$$

meaning the multiplier $\eta$ may have a jump. On the other hand, transition from Cases 2 and 3 to $E$ and $G$ can take place only when

$$
\eta(t)=d \exp (-\gamma t)-\lambda_{w}(t)
$$

is becoming zero and turning positive.

For the infinite time and free terminal state problem possible sequences which are economically meaningful and satisfy all the conditions are

$$
\begin{array}{ll}
B \rightarrow D \rightarrow 1 \rightarrow 3 & \text { or } A \rightarrow 1 \rightarrow 3 \\
A & \text { or } A \rightarrow 2 \rightarrow 3 \\
& \text { or } A \rightarrow 3 .
\end{array}
$$

The trajectory in state space is in Fig. $4 .^{7}$

${ }^{6}$ Several transitions may be ruled out because they would entail violation of inequality constraints.

7 The figures are drawn on the assumption that the ray $D$ is at a lower $r / w[K / W]$ ratio than the ray $F$, so that $D$ intersects $w=1$ to the left of the equilibrium point $r^{*}$. If not, then (even though the state (3) may be physically sustainable) the net contribution of a laborer falls short of the interest return required on the resources required to equip and train him, so perpetual decumulation results. 
This completes the discussion of the necessary conditions. Turning to the question of sufficiency, there are essentially three approaches to the establishment of the optimality of the extremals discussed above. The first approach would use arguments based on the concativity of the system and criterion function and show directly that the stationary extremals are maximizing. The second approach would involve the checking of the Jacobi (conjugate point) condition and the Weierstrass condition along the extremals. Finally, a third approach would be to attempt a numerical solution of the problem using dynamic programming for specific system and criterion functions. This is the particular approach chosen for this problem in view of the analytical complication and the two-dimensional nature of the problem.

A straightforward dynamic programming solution was carried out for

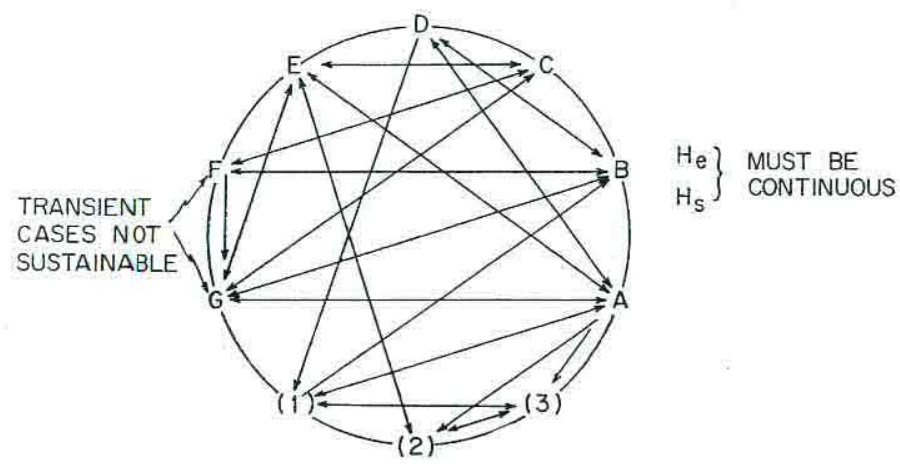

Fig. 3. Possible transition between cases.

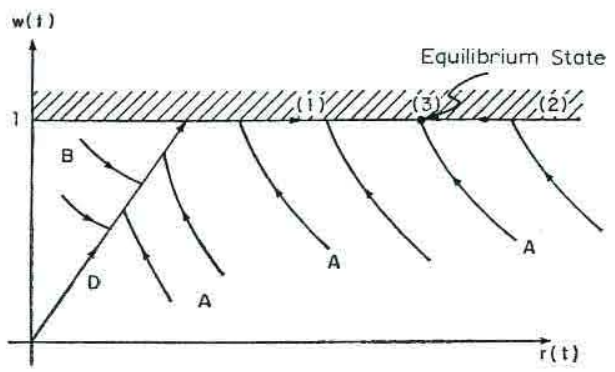

Fig. 4. Qualitative phase trajectories.

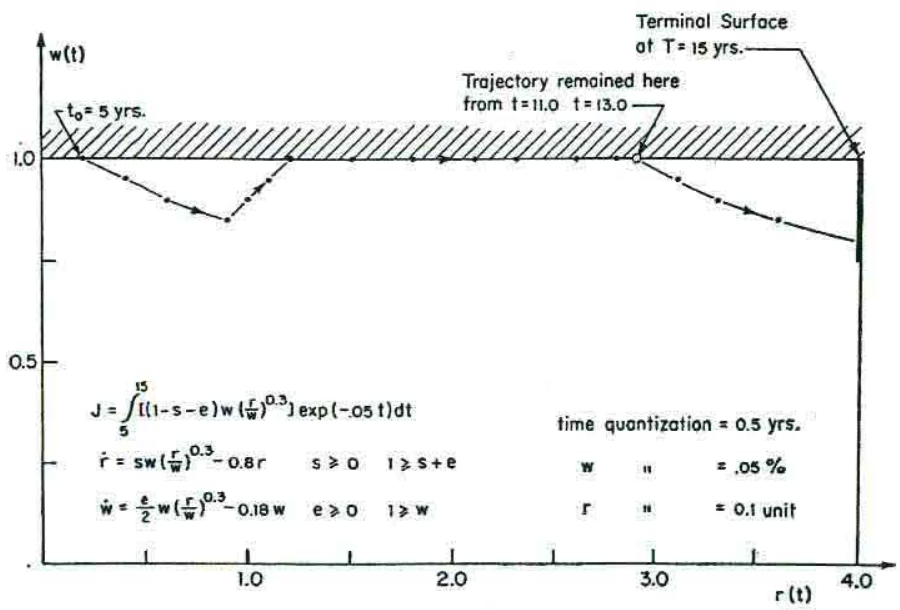

Fig. 5. Optimal trajectory from dynamic programming calculation.

$$
\begin{aligned}
f\left(\frac{r}{w}\right)=\left(\frac{r}{w}\right)^{\alpha} ; \alpha=0.3 ; n & =0.03 \delta=0.05 d=2 \\
\mu & =0.15 \gamma=0.05 .
\end{aligned}
$$

The range of variables involved is

$$
\begin{aligned}
& 0.5 \leq w(t) \leq 1 \quad 10 \text { divisions } \\
& 0 \leq r(t) \leq 4 \quad 40 \text { divisions } \\
& 0 \leq \ell \leq 15=T \quad 30 \text { divisions. } \\
& 0.8 \leq w(T) \leq 1 \\
& r(T)=4
\end{aligned}
$$

First order difference approximations are used for the system differential equations.

A typical trajectory is shown in Fig. 5. It is clear from inspection that it agrees well with the qualitative trajectory obtained in Fig. 4, Section IV.

\section{INTERPRETATION}

It is possible to suggest, without extensive detail, an interesting economic interpretation of the foregoing results. As in mathematical programming or Lagrangian multiplier techniques for constrained extremum problems, the interpretation is based on identifying the $\lambda$ variables as values associated with scarce resources. Consider then, ignoring all that has gone before, tentatively assigning unspecified values $\exp (-\gamma t), \lambda_{r}(t), \lambda_{w}(t)$ as implicit unit prices (in terms of the consumption good at time zero) for the consumption good, the physical capital asset, and the trained laborer, respectively, delivered at time $t .^{8}$

The model economy produces three outputs - a consumption good which is immediately used up, and machines and trained laborers which live on to produce further output. Accepting the assigned prices, the value (scaled to time zero) of the gross national product flow at time $t$ is simply the sum of the product flows, each evaluated at the appropriate discounted price. Denoting this sum $Y$, it becomes

$$
Y=C \exp (-\gamma t)+(K+\delta K) \lambda_{r}+(W+\mu W) \lambda_{w} .
$$

This is the discounted market value of production at time $t$. But it is not quite the proper concept for this economy to employ in its optimizing decisions. The reason is the presence of the full employment (statespace) constraint, which may prevent the economy from producing all the trained labor it wishes, and therefore may, as will be seen below, cause the value $\lambda_{w}$ to diverge from the value which would correctly reflect the net contribution to this society of a trained laborer. To arrive at a corrected measure, it is necessary when the economy is at full employment-that is, when the statespace constraint is binding - to break the value $\lambda_{w}$ in to

${ }^{8}$ The distinction between the same good received at two different times, and the assignment of different values to each, is essential because, it will be recalled, the criterion (12) weights future consumption less heavily, so that all valuations referring to acquisitions at time $t$ must be reduced by the factor $\exp (-\gamma t)$ to reflect value as measured at time zero. It is somewhat more convenient and common in the economics literature to work with undiscounted values by scaling all the above prices by exp $(\gamma t)$. But the scaling convention alters no results, so consistency will be preserved here by continued use of discounted units. 
two components, namely, the discounted cost of training, $d \exp (-\gamma t)$, and the "profit" which makes up the difference between this cost and the market price $\lambda_{w}$. Denoting this "profit" by the symbol $-\eta$, it becomes in the case of full employment,

$$
\lambda_{w}=d \exp (-\gamma t)+(-\eta) .
$$

Below full employment, $\eta$ is assigned the value zero.

This quantity $-\eta$ is in economic terms the capitalized value of the "scarcity rent" due to untrained labor. (For example, if college quarterbacks were scarce, and could be sold to professional teams for $\lambda_{w}$, then $-\eta$ measures the maximum "scholarship" which a high school graduate who could be trained at a cost $d \exp (-\gamma t)$ could extract from a profit-maximizing football coach.) More immediately, $-\eta$ measures the bonus which this model economy could pay to an untrained immigrant (to induce him to enter the country) and still be better off. Suppose, in fact, that all new labor had to be induced to enter in this way. Then the value of gross national product, after deduction of these incentive payments, or rents to untrained labor, would be

$$
\begin{aligned}
Y^{*}= & C \exp (-\gamma t)+(\dot{K}+\delta K) \lambda_{r} \\
& +(W+\mu W)\left(\lambda_{w}+\eta\right) .
\end{aligned}
$$

Substituting from (4) and (6), this may be rewritten

$$
\begin{aligned}
Y^{*}= & {[(1-s-e) \exp (-\gamma t)} \\
& \left.+s \lambda_{r}+\frac{e}{d} \lambda_{\omega}+\frac{e}{d} \eta\right] F(K, W),
\end{aligned}
$$

which is the market value of gross national product except that trained labor is valued at the cost of production rather than the price $\lambda_{w}$. Denoting the expression in square brackets-which is seen to be a weighted average price-by $\alpha^{*}$,

$$
Y^{*}=\alpha^{*} F(K, W) .
$$

Now, since the prices $\lambda_{r}, \lambda_{w}$ purport to measure the value of the durable assets which produce this output, economic theory yields expressions which they ought to satisfy. It can be shown (see Appendix II) that the auxiliary variables $\lambda_{r}, \lambda_{w}$ are the economists' competitive asset prices, and the necessary conditions (38) and (39) are the usual "zero-profit" conditions arising from the requirement that asset prices be the present value of future earnings. ${ }^{9}$ Then, dividing (59) by $L$, it can be seen that the per-capita value of output differs from the Hamiltonian $\mathfrak{H C}$ only by terms which do not involve the controls. Hence, the requirement that the controls be so selected as to maximize $\mathfrak{H}$ has its parallel in the economic

${ }^{9}$ We are grateful to Prof. D. Cass, who discussed this paper at the December, 1965, meetings of the Econometric Society, for several comments. One comment properly emphasized that this interpretation of the multiplier equations is possible only with the linear criterion function employed here. precept that if suitable implicit prices are given, the investment policy which maximizes the implicit value of current output at every instant, also realizes the optimum path over time.

Moreover, it is now easily seen that the catalog of possible cases is based upon a comparison of benefitcost ratios for the production of the three possible outputs. Singular cases arise when these ratios of demand price to cost of production are equal in two or three directions. The last case, in which $\exp (-\gamma t)=\lambda_{r}$ $=\left(\lambda_{w}+\eta\right) / d$, represents the unique equilibrium case, in which the economy has no incentive to alter any allocations.

One further interpretation may be noticed, but will not be pursued here. The condition defining the ray $D$ in Fig. 1 may be written

$$
\alpha^{*}\left[f-\frac{r}{w} f^{\prime}\right]=\left(f^{\prime}+\mu-\delta\right) d \alpha^{*} .
$$

Then, since the left-hand term is the wage rate, and the term $d \alpha^{*}$ is the value of the resources used for training (the training cost), it may be easily checked that an economy which decreed a rate of interest equal to $\left(f^{\prime}+\mu-\delta\right)$ on education loans, and further decreed that untrained labor should be trained when, and only when, the wage level meets the interest charge on an education loan to cover the training cost, would duplicate the optimal trajectories of Fig. 4 until full employment was attained. Moreover, this rule would permit a decentralized policy in that, once the rate was set, each individual could make his own profit maximizing decision, without any need to consult the unknown (and, as mentioned below, generally indeterminate) value $\lambda_{w}$ assigned to an individual laborer.

\section{Conclusions}

The problem studied here is of course highly simplified, and differs relatively little from problems of optimal saving widely discussed in the economics literature [8], [10]. But some of the differences (for example, the existence of state-space constraints and of singular paths which do not represent full system equilibrium) are important enough that a complete and detailed solution to this straightforward problem seems useful, and an essential preliminary to the study of any richer models involving retraining costs or technical progress.

The results of the analysis are for the most part very plausible. Essentially, they require that, except for an initial phase of capital scarcity, the society invest so as to attain full employment as quickly as possible. Other results of interest are, however, obtained along the way, and can be reviewed briefly here.

A planning office, in its wisdom, and with a little computing, can establish the investment allocation rates $s(t), e(t)[0 \leq t \leq T]$ which realize the optimal path of accumulation from any given initial point. These functions represent a solution of the so-called "open loop" control problem. 
Further, the planning office can extend the analysis to an optimal policy $s(r, w), e(r, w)$ which determines the investment allocations at any point in the state space $(r, w)$. Such a policy is a solution of the "feedback control" problem, and permits the system to adjust automatically to shocks along the accumulation path.

To an economist, concerned with decentralized systems, or bound by existing market institutions, still a further form of solution is of interest. The analysis of this essay shows that, if there were observable market prices $\lambda_{r}, \lambda_{w}$, a policy

$$
\begin{aligned}
& s\left(\lambda_{r}, \lambda_{w}\right)=U\left(\lambda_{r}-\frac{\lambda_{w}}{d}\right) U\left(\lambda_{r}-\exp (-\gamma t)\right), \\
& e\left(\lambda_{r}, \lambda_{w}\right)=U\left(\frac{\lambda_{w}}{d}-\lambda_{r}\right) U\left(\frac{\lambda_{w}}{d}-\exp (-\gamma t)\right),
\end{aligned}
$$

where $U$ again represents a unit step function, would be optimal provided $\lambda_{r}, \lambda_{w}$ satisfy the modified zero-profit conditions discussed above, and attain the appropriate terminal values (or, in the infinite horizon case, satisfy the appropriate transversality conditions). The difficult singular cases are taken care of in this rule by allowing some "chatter" in the system, along traditional economic lines. That is, one observes that along the singular paths the allocation according to this policy is indeterminate. If, however, the allocation actually selected should be in error, price adjustments could lead to a nonsingular case with a unique allocation, driving the system back toward the optimal path.

As a final modification of the policy rules, it may be suggested briefly that even if $\lambda_{w}$ should not be observable, there will in some cases be a control law which can be observed by a decentralized system. The existence of such a rule in this case depends on a capital market which provides education loans against future wage income.

The following may also be noted:

1) In general, it is precisely the difficult singular cases in which the economist may frequently be interested, and which will represent the most significant portions of an optimal path.

2) In the presence of a full employment constraint, there will in general be a discontinuity in the implicit value of a trained laborer as full employment is attained. Moreover this implicit value is not determinate in an economy which attains and remains at full employment.

3) The logical possibility of declining employment rates along the optimal path exists, and the quantitative importance of this possibility may be great when physical capital is scarce and training costs high. The allocation decision may be summarized by the requirement that unemployment should rise until the wages of a laborer enable him, in principle, to pay interest at the rate $\left(f^{\prime}+\mu-\delta\right)$ on the cost of training.

4) Neither the cost of training nor the rate of mortality and retraining affects the determination of the opti- mum capital intensity (capital per worker) for a given economy. (In the penalty function approach, these factors determine the appropriate employment rate.)

5) Application of new control theoretic techniques appears useful in the study of this and similar models of investment allocation.

\section{APPENDIX I} Generalized Legendre-Clebsch Test for Cases
$E, D$, and 3

The generalized Legendre-Clebsch test says that for a singular extremal to be optimal (maximal), it must necessarily satisfy the condition that along the singular trajectory

$$
\frac{\partial}{\partial u_{j}} \frac{d^{2}}{d t^{2}} H_{u_{j}} \geq 0
$$

where $u_{j}$ is the particular control variable which is singular. Thus, for Case $\mathrm{E}$ we have

$$
\frac{\partial}{\partial e} \frac{d^{2}}{d t^{2}} H_{e}=\frac{\partial}{\partial e} \frac{d^{2}}{d t^{2}} w f\left(\frac{\lambda_{w}}{d}-\exp (-\gamma t)\right) .
$$

It is laborious but straightforward to show that (2) yields

$$
\frac{\partial}{\partial e} \frac{d^{2}}{d t^{2}} H_{e}=\frac{\partial}{\partial e} w f \frac{d^{2}}{d t^{2}}\left(\frac{\lambda_{w}}{d}-\exp (-\gamma t)\right)
$$

which, after further manipulations, gives

$$
\begin{aligned}
\frac{\partial}{\partial e} \frac{d^{2} H_{e}}{d t^{2}} & =\frac{\partial}{\partial e}\left[\exp (-\gamma t) f^{\prime \prime} \frac{r}{w} \frac{d}{d t}\left(\frac{r}{w}\right)\right] \\
& =\frac{\partial}{\partial e} \exp (-\gamma t) f^{\prime \prime} \frac{r}{w} \cdot \frac{1}{w^{2}}(\dot{r} w-\dot{w} r) \\
& =f^{\prime \prime} \frac{r}{w} \cdot \frac{1}{w^{2}} \exp (-\gamma t)\left(-\frac{r w f}{d}\right) \\
& =-\exp (-\gamma t) f^{\prime \prime} f \frac{r^{2}}{w^{2} d} .
\end{aligned}
$$

Since by assumption $d>0, r>0, w>0, f>0, f^{\prime \prime}<0$, we see that

$$
\frac{\partial}{\partial e} \frac{d^{2}}{d t^{2}} H_{e} \geq 0
$$

as is required. Similarly for Case 3 we have

$$
\begin{aligned}
\frac{\partial}{\partial s} \frac{d^{2}}{d t^{2}} H_{s} & =\frac{\partial}{\partial s} \frac{d^{2}}{d t^{2}}\left(\lambda_{r}-\exp (-\gamma t)\right) \\
& =\frac{\partial}{\partial s} w f \frac{d^{2}}{d t^{2}}\left(\lambda_{r}-\exp (-\gamma t)\right) \\
& =-\frac{\partial}{\partial e} \exp (-\gamma t) f^{\prime \prime} \frac{d}{d i}\left(\frac{r}{w}\right) \\
& =-\exp (-\gamma t) f^{\prime \prime} f \geq 0 .
\end{aligned}
$$


Lastly, for Case D let us use the substitution $s=1-e$ and eliminate $e$. Then the problem is singular in the variable $s$. We have

$$
H_{s}=w f\left(\lambda_{r}-\frac{\lambda_{w}}{d}\right)
$$

and

$$
\begin{aligned}
\frac{\partial}{\partial s} \frac{d^{2}}{d t^{2}} H_{s} & =-w f \frac{w d+r}{w d} \lambda_{r} \frac{\partial}{\partial s} f^{\prime \prime} \frac{d}{d t}\left(\frac{r}{w}\right) \\
& =-w f \frac{w d+r}{w d} \lambda_{r} f^{\prime \prime} f \geq 0
\end{aligned}
$$

as is required.

\section{Appendix II}

Economic Interpretation of $\lambda_{r}$ and $\lambda_{w}$

It is a standard argument that the current earnings of any asset in a competitive economy tend to equality with the marginal product of that asset, which means the earnings of capital goods, properly evaluated, should be

$$
\frac{\partial Y^{*}}{\partial K}=\alpha^{*} \frac{\partial F}{\partial K}=\alpha^{*} f^{\prime}(r / w)
$$

and the earnings (wages) of a trained laborer, properly evaluated, should be

$$
\frac{\partial Y^{*}}{\partial W}=\alpha^{*} \frac{\partial F}{\partial W}=\alpha^{*}\left[f-\frac{r}{w} f^{\prime}\left(\frac{r}{w}\right)\right]
$$

where $\alpha^{*}$ and $Y^{*}$ are as defined in (58) and (60). Further, the price of a durable asset should be the present value of all such current earnings over the lifetime of the asset, future earnings discounted at a suitable rate of discount which reflects physical depreciation as well as market interest rates. Taking the market rate of interest to be $n$, the price of a unit of the capital good would be calculated, in economic theory, as

$$
\lambda_{r}=\int_{t}^{\infty} \exp [-(n+\delta)(\tau-t)] \alpha^{*} f^{\prime}\left(\frac{r}{\varkappa}\right) d \tau .
$$

Differentiating with respect to $t$, and comparing the result with (38), one sees that the auxiliary variable $\lambda_{r}$ defined by (38) together with the terminal condition (56), is exactly the price which would be computed for capital goods from economic theory if a market rate $n$ were used in discounting future earnings. (It should be noted that all these prices, including $\alpha^{*}$, are already discounted by the factor $\exp (-\gamma t)$. Working in current, undiscounted values, one could take $n+\gamma$ as the market rate.) Were it not for the unearned scarcity rents accruing to labor, a parallel argument would suffice to interpret $\lambda_{w}$. But here the initial bonus must be accounted for in the economic pricing relationships, which essentially require that values be conserved. One might conceive of the accounting procedure as assigning the scarcity value $-\eta(t)$ to laborers at any time $t$, and paying interest on this amount to each laborer as a supplement to his earnings given in (69). Then the value $\lambda_{w}$ would be the capitalized value of earned and unearned incomes, given by

$$
\begin{aligned}
\lambda_{w}= & \int_{t}^{\infty} \exp [-(n+\mu)(\tau-t)]\left[\alpha^{*}\left(f-(r / w) f^{\prime}(r / w)\right)\right. \\
& -(n+\mu) \eta] d \tau .
\end{aligned}
$$

Differentiating this expression with respect to $t$, and comparing it with (39), one sees that the auxiliary variable $\lambda_{w}$ defined by (39) agrees with this implicit price of trained labor, up to a possible constant difference. It appears, however, that as long as this economy remains at full employment, there is insufficient information to determine $\lambda_{w}$ and $\eta$ separately. Setting $-\eta \equiv M>0$ after full employment is attained leads to

$$
\begin{aligned}
& \lambda_{w}=\int_{t}^{\infty} \exp [-(n+\mu)(\tau-t)] \alpha^{*}\left[f-(r / w) f^{\prime}\right] d \tau+M \\
& \lambda_{w}+\eta=d \exp (-\gamma t) \\
& \quad=\int_{t}^{\infty} \exp [-(n+\mu)(\tau-t)] \alpha^{*}\left[f-(r / w) f^{\prime}\right] d \tau,
\end{aligned}
$$

which agrees with economic intuition, and

$$
\lambda_{v}+\eta \rightarrow 0 \quad \text { as } t \rightarrow \infty,
$$

so that (56) is satisfied. The arbitrariness of the value of $\eta$ again agrees with results from control theory [1].

\section{ACKNOWLEDGMENT}

The authors wish to thank D. Bailin and J. Daniels for their assistance in computation.

\section{REFERENCES}

[1] A. E. Bryson, W. F. Denham, and S. E. Dreyfus, "Optimal programming problems with inequality constraints. I: Necessary conditions for extremal solutions," J. AIAA, vol. 1, pp. 25442550, November 1963.

[2] A. E. Bryson and Y. C. Ho, Optimal Programming, Estimation, and Conirol. New York: Blaisdell (to be published).

[3] D. Cass, "Optimum growth in an aggregative model of capital accumulation," Rev. Econ. Studies, vol. 32, pp. 233-240, October 1965.

[4] S. E. Dreyfus, Dynamic Programming and the Calculus of Variations. New York: Academic Press, 1965.

[5] H. J. Kelley, R. Kopp, and G. Moyer, "Singular extremals in optimal control," in Optimization Techniques, vol. 2, G. Leitman, Ed. New York: Academic Press (to be published).

[6] T. C. Koopmans, "On the concept of optimal economic growth," Cowles Foundation, Yale University, Discussion Paper 163, 1963.

[7] L. S. Pontryagin et al., The Mathematical Theory of Optimal Processes, translated by K. N. Trirogoff. New York: Interscience, 1962

[8] F. P. Ramsey, "A mathematical theory of saving," Economic J., vol. 38 , pp. $543-559,1928$.

[9] P. A. Samuelson, "A catenary theorem involving consumption and the Golden Rule," Am. Econ. Rev., pp. 486-496, June 1965.

[10] P. A. Samuelson and R. M. Solow, "A complete capital model involving heterogeneous capital goods," Quarterly J. Econ., vol. 70 , pp. 537-562, November 1956.

[11] K. Shell, "Optimal programs of capital accumulation for an economy in which there is exogenous technical change," Mass. Inst. Tech., Cambridge. Mass., Discussion Paper, undated.

[12] K. S. Tait, "A generalized Legendre condition for singular optimal control problems," Ph.D. dissertation, Harvard University, Cambridge, Mass., 1965 (unpublished). 